protein particles out of the nucleus, including ribosomes and messenger RNA. This process is also highly selective, resulting in the export of mature transcripts but the retention in the nucleus of immatuie transcripts. The basis of this selectivity is unknown, but in at least one case there is evidence of a carrier mediated transport system.

\section{Rebuilding the cell nucleus}

Recently there have been substantial advances in our ability to reconstruct "nuclei" from condensed chromatin or even purified DNA in cell free systems. Figure 3 shows a large fully enveloped nucleus which has been formed from demembranated frog sperm chromatin in a cell free system derived from frog eggs. Similar structures have now been formed from purified DNA, including DNA from bacterial viruses and plasmids. Not only is such DNA assembled into chromatin; it also becomes surrounded by nuclear membranes containing functional nuclear pores. Thus these pseudonuclei fill up with nuclear proteins and even replicate their DNA in vitro.

The opportunity to reconstruct functioning nuclei and functioning chromosomes opens up new approaches to understanding the complex structure and function of the cell nucleus.
Further reading

General

Alberts B, Bray D, Lewis J, Raff M, Roberts K, Watson JD. Molecular biology of the ceil. Chapter 8. New York and London: Garland Inc, 1983.

Lewin B. Genes III. New York: Wiley, 1987.

Chromatin and chromosomes

Blackburn EM. Artificial chromosomes in yeast. Trends in Genetics 1985;1:8-12.

Earnshaw WC, Laemmli UK. Architecture of metaphase chromosomes and chromosome scaffolds. J Cell Biol 1983;96:84-93.

Klug A, Rhodes D, Smith J, Finch JT, Thomas JO. A low resolution structure for the histone core of the nucleosome. Nature 1980;287:509-16.

Gene expression

Dynan WS, Tjian R. Control of eukaryotic messenger RNA synthesis by sequence-specific DNA binding proteins. Nature 1985;316:774-8. binding proteins. Nature 1985;316:774-8.
Serfling E, Jasin M, Schaffner W. Enhancers and eukaryotic gene transcription. Trends in Genetics
1985;1:224-30.

Harrison SC. Fingers and DNA half turns. Nature 1986;322:597-8.

Role of the nuclear envelope

Dingwall C. The accumulation of proteins in the nucleus. Trends in Biochemical Sciences 1985;10:64-6. Rebuilding the cell nucleus

Blow JJ, Laskey RA. Initiation of DNA replication in nuclei and purified DNA by a cell-free extract of Xenopus eggs. Cell 1986;47:577-87.

Laskey RA. Prospects for reassembling the cell nucleus. f Cell Sci [Suppl] 1986;4:1-9.

\title{
Epidemiology
}

\section{Report from the PHLS Communicable Disease Surveillance Centre}

During the summer the epidemic of meningococcal meningitis receded, with notifications falling to their 1986 level, though a few infections were imported from Saudi Arabia; laboratory reports of respiratory infections due to parainfluenza type 3 rose, while those due to respiratory syncytial virus and Mycoplasma pneumoniae declined; there was an outbreak of campylobacter infection due to pasteurised milk, and the number of laboratory reports of both campylobacter and salmonellosis was higher than ever before for the same period of the year; and the cumulative total of cases of the acquired immune deficiency syndrome (AIDS) reported in the UK reached over 1000 by the end of August.

\section{Meningococcal meningitis}

Over 400 cases of meningococcal meningitis were notified in the first quarter of 1987 in England and Wales, almost twice the number seen in the same period in 1986 and about the same as the peak of the previous epidemic in 1974. Laboratory reports of meningococcal bacteraemia and meningitis showed the same trend, but, unlike the rise in 1985 and 1986, which was associated with an increase in reports of group B type 15 strains of Neisseria meningitidis, notably from the Gloucester area, the rise seen in 1987 was mainly due to group C strains. A seasonal fall was expected during the spring and summer, but notifications were thought likely to continue at about double the 1986 level. Surprisingly, however, the epidemic receded, notifications falling back to almost the same as 1986, closely resembling the pattern of the previous epidemic in 1974 (fig 1). If this pattern is followed in the next three years a further

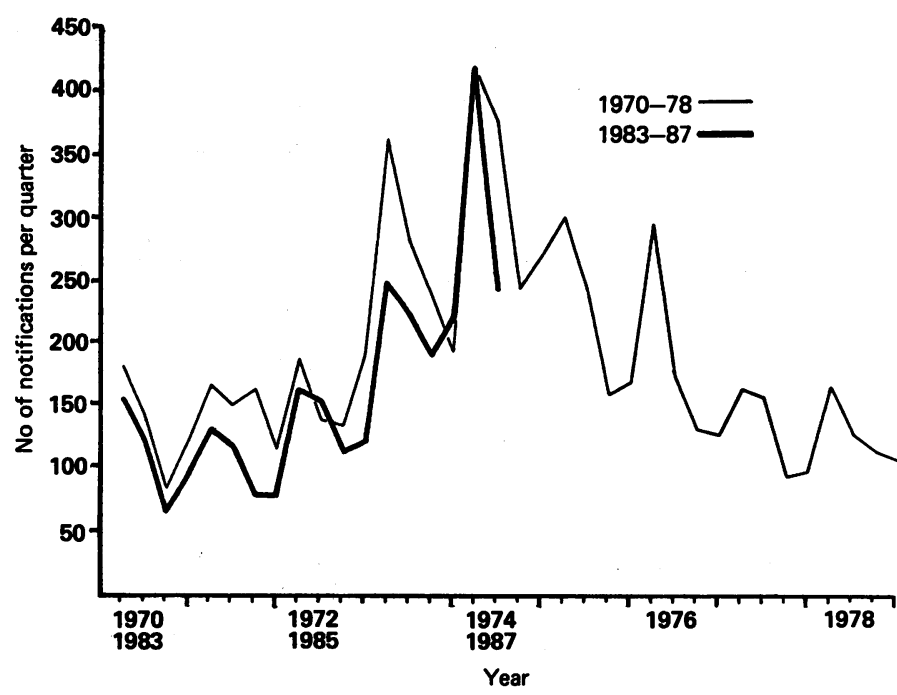

FIG 1-Meningococcal meningitis in England and Wales 1970-8 and 1983-7.

fall in notifications would be expected, reaching "normal" interepidemic levels in 1990 . Even so, there will still probably be at least 400 notifications of meningococcal meningitis a year and as many cases of meningococcal systemic infections with or without meningitis which escape notification and about 80 registered deaths, over half of them in people aged under 20 years.

A less common event took place in August, when $N$ meningitidis 
group A infections were reported in pilgrims returning from Mecca and in their close contacts. This strain was highly resistant to sulphonamides, and rifampicin was therefore recommended for chemoprophylaxis in household contacts. Anecdotal reports indicate a high incidence of the infection in the Arabian peninsular, so vaccination should be considered for people visiting Saudi Arabia and the Gulf states who are likely to live or work in overcrowded conditions, such as occurred during the pilgrimage, or who will be working in the health professions.

Future trends in the incidence of meningococcal infection are unpredictable, but the disease is likely to remain an important cause of death in the young. Clinicians should be alert to the possibility of meningococcal disease and should start penicillin immediately rather than delay this until admission to hospital, especially in patients with septicaemia presenting with purpuric rash without meningitis.

\section{Respiratory infections}

During the summer the number of laboratory reports of $M y c 0-$ plasma pneumoniae infections declined as expected from a peak earlier in the year. The incidence is likely to be low over the next two years until the winter of 1989-90, when the next epidemic will probably begin and reach a peak in early 1991. This regular four yearly cycle has been evident since the early 1970 s, when laboratory diagnosis of the infection began to be widely used (fig 2 ).
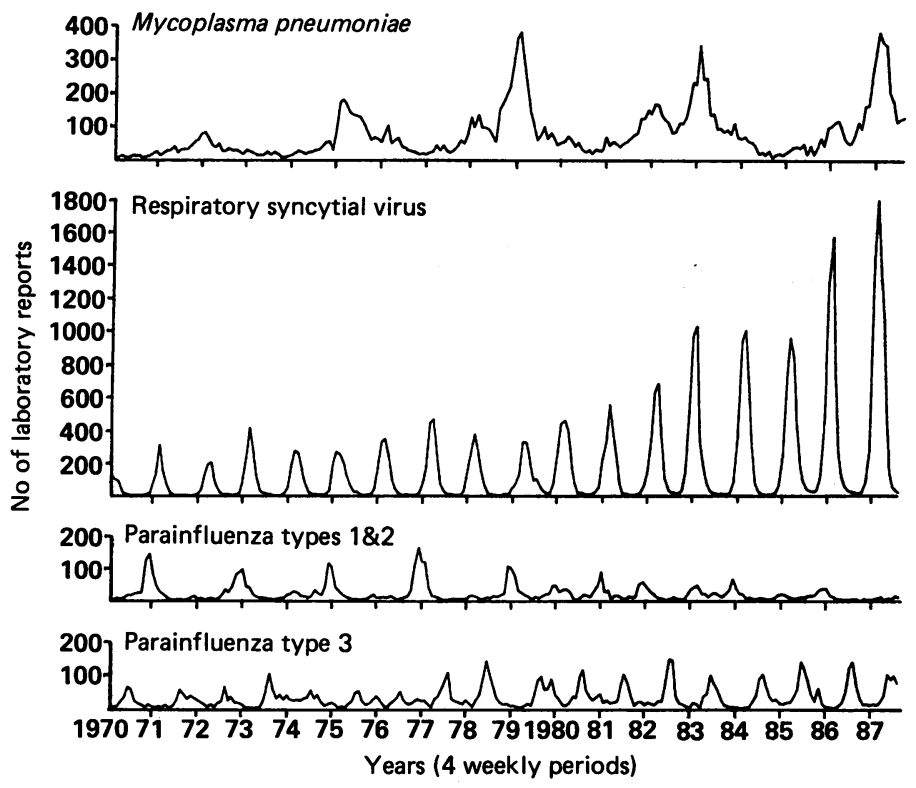

FIG 2-Respiratory infections in England and Wales 1970-87.

Laboratory reports of respiratory syncytial virus infection have also maintained a regular pattern since the early 1970s but follow an annual cycle. This year the winter peak occurred in January, when there were nearly 1800 reports, but numbers fell rapidly to almost nil during the summer. The greater height of the peak this year (fig 2) was probably an artefact caused by more widely available laboratory diagnostic tests and increased laboratory reporting.

As the number of laboratory reports of $M$ pneumoniae and respiratory syncytial virus infections declined, those due to parainfluenza type 3 increased and became widespread, reaching a peak during the summer months. This virus, which commonly causes croup, bronchitis, and pneumonia in young children, also shows a seasonal pattern with a summer peak. In contrast, parainfluenza virus types 1 and 2 showed a regular two yearly cycle with epidemics in alternate winters until the early 1980 s, when the pattern became irregular (fig 2).

\section{Salmonellosis}

In the first half of 1987 there were over 5000 laboratory reports of salmonellosis, the highest number ever recorded in the first half of the year. Salmonellas, other than Salmonella typhi and $S$ paratyphi, are the commonest cause of bacterial food poisoning, so trends in laboratory reports of this organism closely follow trends in statutory notifications of food poisoning; there were nearly 8000 notifications in the first half of 1987, again higher than ever before.

The numbers of cases of salmonellosis and food poisoning have increased almost continuously since the Public Health Laboratory Service began surveillance of foodborne disease in the early 1940s. Some of this increase is almost certainly due to the wider availability of laboratory diagnosis, increased interest in the disease, more detailed investigation of suspected food poisoning, and better reporting. Nevertheless, most of the increase is probably due to a real rise in the incidence of salmonellosis because there have been wide variations in the incidence of different serotypes over time; the most recent rise, due mainly to a steep rise in cases of $S$ typhimurium infection, has not been adequately explained.

Salmonellosis is often mistakenly regarded as a mild diarrhoeal illness by those who do not appreciate that serious extraintestinal complications may occur, particularly in the very young, the elderly, and other vulnerable groups-for example, those who have undergone gastric surgery. In the 10 years $1975-84$, of 100000 reported infections, 50 were cases of salmonella meningitis, 1367 cases of bacteraemia, and 84 cases of osteomyelitis or septic arthritis. In the same 10 years there were 443 registered deaths due to salmonellosis and many more in which it contributed to death.

Clinicians report cases of salmonellosis informally at an early stage to their community physician colleagues so prompt epidemiological investigations may be made to discover the causes of this increasing problem.

\section{Campylobacter infection}

Unlike salmonellosis, campylobacter enteritis is self limiting, and serious complications are very rare. It was recognised only 10 years ago as a common gastrointestinal infection in humans, since when the number of laboratory reported infections has risen continuously; it is now the most frequently identified cause of acute gastrointestinal illness in Britain with more than double the number of laboratory reported infections than of salmonellosis each year. In the first half of 1987 there were over 11000 reports, again the highest number ever recorded in the first half of the year.

Campylobacter enteritis is usually a foodborne infection, the organism originating from food animals or their environment. Human excretors of the organism do not seem to play a part in disseminating the disease. Evidence from several countries suggests that most cases are probably associated with handling or eating fresh poultry, although this has yet to be shown in Britain. Milkborne disease has been frequently reported; in England and Wales in 1978-86 there were 40 recorded milkborne outbreaks with at least 5156 cases, although these were only a small proportion of the nearly 137000 cases reported in this period. In the first half of 1987 several suspected milkborne outbreaks were investigated; one, at Braintree, which affected over 180 people, was of particular interest because the infection appeared to be conveyed by pasteurised milk.

\section{Pasteurised milk and infection}

Infections due to pasteurised milk are exceptional and when they have occurred have usually been due to contamination after pasteurisation - for example, by milk bottles "cleaned" by washing in contaminated water. A recent review by the Communicable Diseases Surveillance Centre (CDSC) of milkborne disease in England and Wales showed that there were four recorded outbreaks of illness due to pasteurised milk in the 40 years 1938-78, but from 1979 to mid-1987 there were six such outbreaks, two of salmonellosis and four of campylobacter infection, including the Braintree 
outbreak. It is difficult to determine whether this increase is due to improved detection of milkborne disease or to a real increase in outbreaks of infection caused by pasteurised milk. Whatever the explanation, these outbreaks are worrying and emphasise the need for expert supervision and constant vigilance at pasteurisation plants and at all stages of production and distribution of milk and milk products.

\section{Acquired immune deficiency syndrome}

By the end of August 1987 the cumulative total of reported cases of AIDS in the UK since the epidemic began was 1013 with 572 deaths. The distribution by transmission characteristic remained unchanged, with $85 \%$ of cases in homosexual or bisexual men. Comparison of these data, derived from clinical reports to the CDSC and the Communicable Diseases (Scotland) Unit $(\mathrm{CD}(\mathrm{S}) \mathrm{U})$, with national mortality data and with locally available information suggests that clinical reporting is incomplete. It is particularly important to ascertain the true size of the epidemic and its development over time and by risk group. Clinicians are therefore asked to ensure that all their cases or suspected cases are reported as soon as possible to either the CDSC (61 Colindale Avenue, London NW9 5EQ, Tel: 01-200 6868) or the CD(S)U (Ruchill Hospital, Glasgow G20 9NB, Tel: 041-946 7120). A revised report form with a tear off front page for local use is obtainable from either centre.

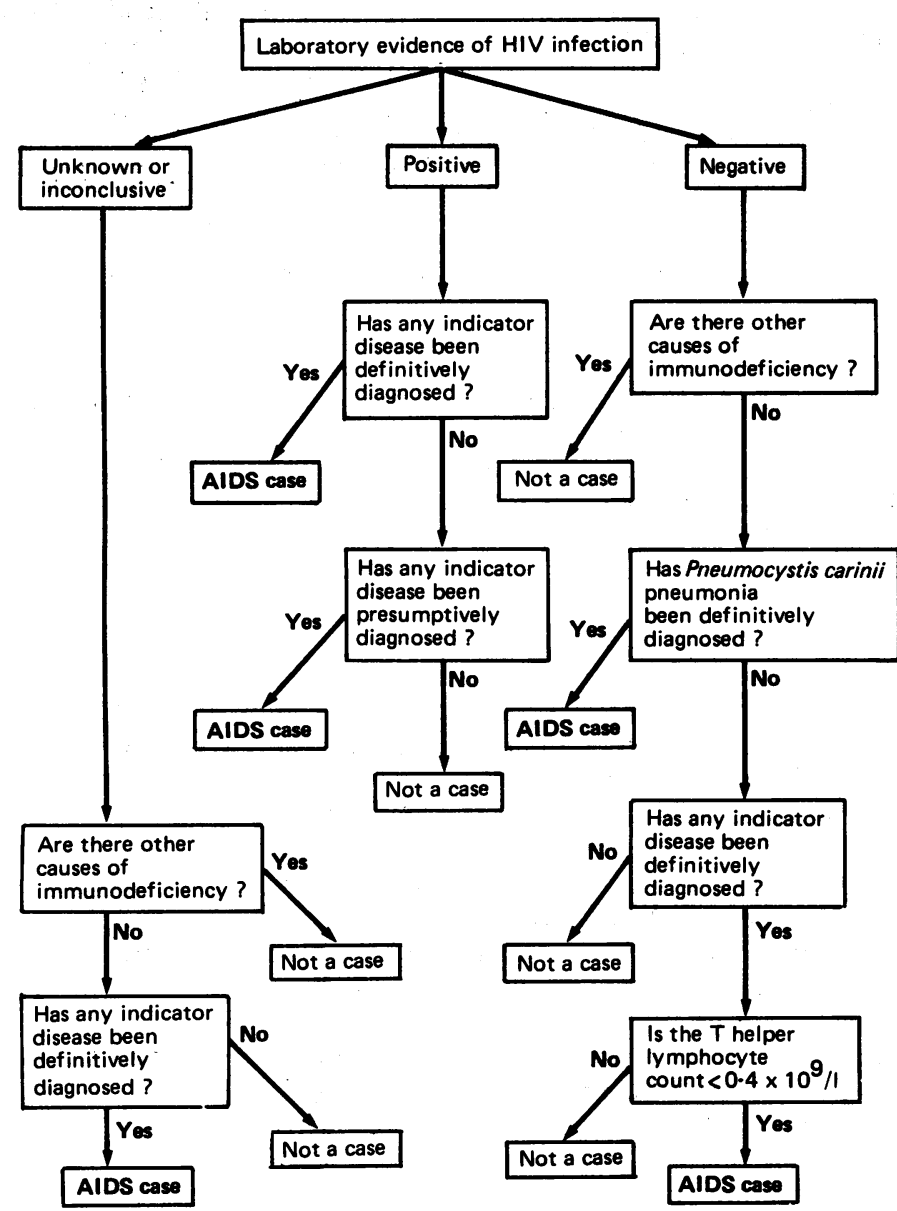

FIG 3-Flow diagram for revised case definition of AIDS (based on the diagram published in $M M W R$ 1987;36(suppl 1S)).
Paediatricians should report cases of AIDS in children to the British Paediatric Surveillance Unit, after which they are followed up by the CDSC or the $\mathrm{CD}(\mathrm{S}) \mathrm{U}$. Similarly, haemophilia physicians should report all cases of AIDS and disease related to the human immunodeficiency virus (HIV) to the Oxford Haemophilia Centre, where the data required by $\mathrm{CDSC}$ and $\mathrm{CD}(\mathrm{S}) \mathrm{U}$ will be recorded without patient names and further information obtained from the reporting clinician if necessary.

\section{REVISED CASE DEFINITION OF AIDS}

A revised definition of AIDS has been published by the Centers for Disease Control, Atlanta, ${ }^{1}$ and will be used by the CDSC and the $\mathrm{CD}(\mathrm{S}) \mathrm{U}$ from 1 September 1987. This new definition includes HIV encephalopathy, HIV wasting syndrome, and a broader range of AIDS indicator diseases. It also permits, in certain circumstances, the inclusion of cases without evidence of HIV infection or with laboratory evidence against infection.

The definition is in three sections, according to the status of laboratory evidence of HIV infection.

Without evidence of HIV infection (no test done or inconclusive results): a definitively diagnosed specified indicator disease and no other cause of immunodeficiency.

With laboratory evidence of HIV infection, regardless of the presence of other causes of immunodeficiency: any of the specified indicator diseases whether diagnosed definitively or presumptively.

With laboratory evidence against HIV infection and no other cause of immunodeficiency: either definitively diagnosed Pneumocystis carinii pneumonia, or both a specified definitively diagnosed indicator disease plus a T helper/inducer cell count below $0.4 \times 10^{\%} / 1$.

The Centers for Disease Control document includes a flow diagram to help in assessing cases in these three categories (fig 3).

The definition for children differs from that for adults in two ways: $(a)$ multiple or recurrent serious bacterial infections and lymhoid interstitial pneumonia or pulmonary lymphoid hyperplasia are accepted as indicative of AIDS among children; and (b) laboratory criteria for HIV infection are more stringent for children aged 15 months whose mothers are thought to have had HIV infection during the child's perinatal period.

Copies of the document can be obtained from: Superintendent of Documents, US Government Printing Office, Washington DC 20402-9325, USA, price $\$ 1.25$ (includes postage to UK).

"Diet" drinks usually contain artificial sweeteners. Is there any evidence that such sweeteners are carcinogenic?

The three artificial sweeteners in common use are aspartame (Nutrasweet), cyclamate, and saccharin. Most diet drinks contain mainly aspartame as the sweetener, though often with small amounts of saccharin or cyclamate as well. Aspartame is rapidly broken down in the body to its two constituent amino acids phenylalanine and aspartic acid. Carcinogenicity would therefore not be expected and has not been found. Cyclamate and saccharin have both been suspected of being carcinogenic on the basis of animal data; large doses were found to produce bladder tumours in some animal species. There is no evidence that this is relevant to their use in man and the suspicion has now been largely dismissed. In 1967 the World Health Organisation put a safety limit on cyclamate of $50 \mathrm{mg} / \mathrm{kg}$, and this could be exceeded if large quantities of soft drinks sweetened entirely with cyclamate were consumed. It is unlikely that this could occur with the diet drinks available now.LINDA BEELEY, consultant clinical pharmacologist, Birmingham.

Fastner Z. Miscellaneous drugs. In: Dukes MNG, ed. Meyler's side effects of drugs. 10th ed. Amsterdam, New York, Oxford: Elsevier, 1984:913. 